\title{
Problèmes associés à l'étude du bruit d'un circuit de pompes centrifuges
}

\author{
Problems associated with the investigation of \\ centrifugal pump noise
}

\author{
Jean Tourret \\ Responsable du Secteur Acoustique du CETIM (Senlis)
}

\section{Introduction}

Cet exposé aborde certains problèmes posés par la mesure et la prévision du bruit des pompes centrifuges. Ces machines, dont l'éventail s'étend des circulateurs de chauffage central aux pompes alimentaires des chaudières de centrales, posent à des titres divers des problèmes de bruit.

S'il est généralement difficile de prévoir le niveau de bruit des nouvelles machines, il faut aussi reconnaitre que la qualité des mesures de puissance acoustique (fréquemment conduites lors des essais de réception hydraulique sur plate-forme ou sur le site de fonctionnement) peut laisser perplexe (bruit de fond important, réverbération, moteur d'entraînement directement accouplé, proximité de la vanne de réglage du débit parfois beaucoup plus bruyante, mauvaise adaptation des tuyauteries, résonances...).

Après une définition des phénomènes acoustiques et vibratoires rencontrés dans les installations hydrauliques, nous aborderons succinctement les problèmes de bruit posés par les éléments des circuits puis nous nous attacherons plus particulièrement à l'étude d'une pompe centrifuge de taille moyenne.

Les phénomènes acoustiques et vibratoires rencontrés dans les installations hydrauliques et leur mesure

Il ne peut être question de rappeler ici les notions élémentaires d'acoustique que l'on rencontrera dans les pages suivantes et l'on pourra se reporter si nécessaire aux références citées dans [1] qui contient les définitions de base.

Les phénomènes vibratoires ont pour origine les mouvements relatifs des structures mécaniques et du fluide, ainsi que ceux des structures mécaniques entre elles. L'énergie développée en un point est transférée sous diverses formes d'ondes (vibrations longitudinales, transversales, de flexion ou de torsion) à travers les différents milieux et avec des efficacités très variables dues en particulier aux phénomènes d'atténuation et de réflexion ; l'énergie sonore est finalement rayonnée par les surfaces externes en contact avec l'air (carters, volute de pompe, corps de vannes, canalisations, châssis, fondations...).

L'étude du rayonnement acoustique d'un élément de structure mécanique appartenant à une installation hydraulique passe par la connaissance des trois types d'information suivante :

a) Distribution temporelle des forces excitatrices en tout point de la structure mécanique envisagée.

Ces excitations peuvent être appliquées :

- par l'intermédiaire d'autres éléments connectés à l'élément mécanique considéré (ce qui nécessite la connaissance des impédances mécaniques sur les frontières de raccordement),

- par le liquide lui-même, siège de fluctuations de pression qui entraînent des fluctuations de force sur toute paroi qui limite le fluide.

b) Réponse des surfaces en contact avec l'air aux excitations fournies à la structure

Celle-ci peut théoriquement être décrite, pour tout couple de points et pour chaque valeur de la fréquence, sous forme d'admittance comme quotient de la vitesse vibratoire en un point par la force excitatrice en un autre point. Une telle grandeur est caractérisée par un nombre plus ou moins grand de pics de résonance pouvant être très rapprochés et qui indiquent les différents modes de vibrations de la structure; ceux-ci sont conditionnés par la distribution géométrique des masses et raideurs, et dans une certaine mesure par la nature de l'amortissement ("proportionnel" ou non). 
Dans certains cas pratiques, on est conduit à considérer par simplification [2] une admittance moyenne entre les vitesses vibratoires moyennées sur une surface et la force excitatrice en un point ou un ensemble de points, grandeur qui fait alors abstraction des phénomènes de résonance.

\section{c) Coefficient de rayonnement des surfaces en contact avec l'air}

Les mouvements périodiques d'une surface en contact avec l'air mettent en vibrations les molécules voisines à la même vitesse que cette surface. La détermination du rayonnement acoustique d'une structure à partir de la connaissance de l'état vibratoire de sa surface est un problème complexe dont la solution dépend en particulier de la géométrie des surfaces mises en jeu, ainsi que des domaines de fréquences concernés. On définit un coefficient de rayonnement qui est le rapport de la puissance acoustique rayonnée par un élément de surface (appartenant à une structure mécanique) à la puissance acoustique rayonnée par un piston rigide de même surface disposé dans un baffle. Ce coefficient possède généralement une valeur d'abord croissante avec la fréquence et tendant ensuite vers l'unité.

A la connaissance de ces trois types d'informations, il convient d'ajouter celle qui concerne la sensibilité de l'oreille et qui est généralement fournie par la courbe de pondération $A$ [1]. On peut alors relier le niveau sonore perçu par l'oreille aux mécanismes d'excitation à l'aide d'une "fonction globale de transmission" dépendant de la fréquence :

$\left[\begin{array}{c}\text { fonction globale } \\ \text { de transmission }\end{array}\right]=[$ admittance $] \times\left[\begin{array}{c}\text { degré de } \\ \text { rayonnement }\end{array}\right] \times\left[\begin{array}{c}\text { pondération } \\ (\mathrm{A})\end{array}\right]$

Toute action de réduction de bruit doit donc tendre à la fois à réduire la fonction globale de transmission et les excitations.

Le technicien qui se trouve confronté à un problème de bruit dans une installation de pompage doit de ce fait recueillir, à l'aiđe de plusieurs types de capteurs des informations sur différentes grandeurs :

- la pression acoustique qui est parfois qualifiée de "bruit aérien" ("airborne noise" dans la littérature anglaise) est mesurée à l'aide d'un microphone ; l'unité correspondante est le décibel (dB) exprimé sous la forme du rapport logarithmique

$20 \log p / p_{0}$ par rapport à la référence $p_{0}=2.10^{-5}$ pascal $\left(1\right.$ pascal $=1$ newton $\left./ \mathrm{m}^{2}\right)$;

- les vibrations de structure parfois qualifiées de "bruit de structure" ("structure borne sound") lorsqu'elles intéressent la gamme des fréquences audibles, sont mesurées en un point à l'aide d'accéléromètres ou de capteurs de vitesse vibratoire ; les niveaux correspondants s'exprimant assez généralement en décibels par rapport à une accélération $\left(\gamma_{0}=10^{-5} \mathrm{~m} / \mathrm{s}^{2}\right)$ ou une vitesse $\left(v_{0}=10^{-8} \mathrm{~m} / \mathrm{s}\right)$ de référence ;

- les fluctuations de pression au sein du liquide ou "bruit hydraulique" ("fluid borne noise") correspondent aux variations instantanées de la pression statique du liquide ; elles sont mesurées dans les veines fluides des machines ou dans les tuyauteries, à l'aide de capteurs de pression à membrane affleu- rante (de type piézoélectrique ou piézorésistif) ou au moyen d'hydrophones qui sont plus sensibles, les niveaux correspondants étant couramment exprimées en décibels par rapport à la référence $p_{0}=0,1 \mathrm{~Pa}$ (= 1 microbar).

Les différents signaux obtenus sont analysés, en fréquences, soit en bandes fines, soit en tiers d'octave ou octave [1] ; ces analyses renseignent sur la répartition spectrale des énergies et par là-même sur les mécanismes de génération et de transfert. A titre d'illustration, les figures $1 \mathrm{a}$ et $1 \mathrm{~b}$ montrent les analyses fines et tiers d'octave des signaux de fluctuations de pression, mesurées dans la tuyauterie de refoulement d'une pompe centrifuge. On note que ces spectres comportent une énergie répartie sur toutes les fréquences à laquelle se superposent des raies (multiples de la fréquence de rotation - ici $50 \mathrm{~Hz}$ ).
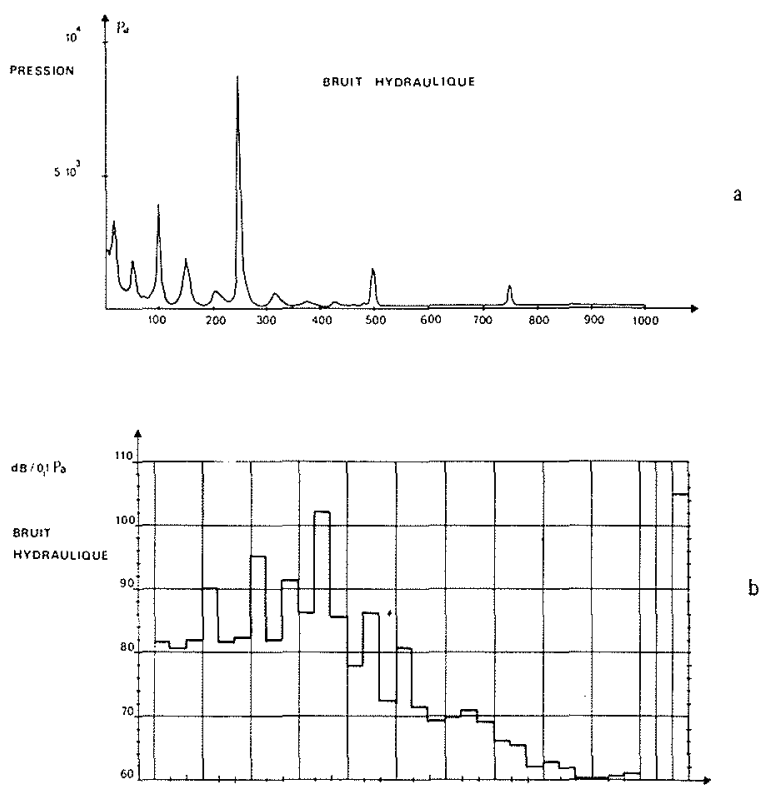

Figure 1 - Analyse des signaux de fluctuations de pression mesurés dans la tuyauterie de refoulement d'une pompe centrifuge. La figure a représente l'analyse fine sur $0-1 \mathrm{kHz}$.

La figure b représente l'analyse tiers d'octave, correspondant à des conditions de fonctionnement voisines.

L'analyse spectrale peut être répétée pour chaque régime de fonctionnement et multipliée un grand nombre de fois en discrétisant finement l'évolution du régime (analyse tridimensionnelle). Une telle méthode inspirée des sonagrammes classiques a été mise au point dans le cadre de cette étude ; elle effectue la synthèse des analyses en tiers d'octave ou en bande étroite par une représentation des niveaux au moyen de 3 couleurs possédant des densités variables d'écriture [3] et permet en particulier de mettre en évidence l'évolution des composantes spectrales et les phénomènes de résonance (voir fig. 7) (*).

(*) Document disponible : Secteur Acoustique - CETIM BP 6760304 Senlis. 
Les mécanismes de génération de bruit dans les pompes centrifuges

$\mathrm{Si}$ les sources mécaniques (paliers, roulements) ne sont généralement pas négligeables -on peut par exemple s'en faire une idée en faisant tourner la pompe dénoyée en lubrifiant les paliers mais en négligeant toutefois les effets de poussée axiale - le bruit de ce type de machine est essentiellement associé à des mécanismes de génération hydrauliques périodiques ou aléatoires.

Les phénomènes périodiques correspondent au passage des aubes mobiles devant le bec de volute ou les aubes fixes du diffuseur et donnent naissance à un spectre de raies où l'on retrouve en particulier :

- la fréquence de rotation $\Omega$

- la fréquence de passage des aubes $N_{R} \cdot \Omega$

- la fréquence de coïncidence des aubes

$N_{R} . N_{S} . /$ P.G.C.D. $\left(N_{R}, N_{S}\right)$

- les harmoniques de ces différentes fréquences

avec $N_{R}$ : nombre d'aubes de la roue,

$N_{S}$ : nombre d'aubes du diffuseur

P.G.C.D. : plus grand commun diviseur

Les phénomènes aléatoires sont dus à la turbulence ou à la cavitation ; ils donnent naissance à une énergie acoustique répartie sur toutes les fréquences qui dans certaines conditions de fonctionnement peut être bien supérieure à celle qui est associée aux phénomènes périodiques.

Ces mécanismes de génération de bruit étant étroitement liés à la transformation d'énergie mécanique en énergie d'écoulement, ils sont associés de façon similaire aux facteurs qui régissent ce processus, à savoir :

- les paramètres géométriques de la machine (tracé de la volute et du diffuseur éventuel, diamètre de l'impulseur, nombre d'aubes mobiles et fixes, profil des aubes...)

- les conditions de fonctionnement (vitesse de rotation $\Omega$, hauteur manométrique $\Delta H$, débit $Q$, N.P.S.H. disponible...).

Dans la plupart des machines, les phénomènes de turbulence et de cavitation sont moindres au point de rendement optimal et le bruit est principalement dû aux composantes périodiques; c'est pourquoi certaines études théoriques [5] se sont attachées au calcul du champ de pression périodique dans la volute. Ces travaux ont d'ailleurs abouti à l'établissement de relations simplifiées semi-empiriques permettant de déterminer à la fois le niveau de bruit hydraulique à caractère périodique (délivré dans la ligne de refoulement où il est très généralement supérieur à celui qui est délivré à l'aspiration) et le niveau de bruit aérien correspondant, en fonction de paramètres géométriques et hydrauliques [6]

- niveau de bruit hydraulique $=$

$$
\begin{aligned}
- \text { niveau de bruit aérien }= \\
\\
\qquad K_{2}+10 \log \mathrm{Q} \Delta H \Omega\left(\mathrm{dB} / 2 \cdot 10^{-5} \text { pascal }\right)
\end{aligned}
$$$$
K_{1}+20 \log \frac{Q \cdot \Delta H}{N_{S} \Omega R^{2} t}(\mathrm{~dB} / 0,1 \text { pascal })
$$

avec:

$K_{1}, K_{2}$ : constantes dépendant du système d'unités et du type de machine

$Q . \Delta H$ : puissance hydraulique
$N_{S} \quad$ vitesse spécifique $\infty \frac{\Omega Q^{1 / 2}}{(\Delta H)^{3 / 4}}$

$R, t \quad$ rayon de l'impulseur et largeur à son extrémité $\Omega \quad$ vitesse de rotation

Ces relations permettent en particulier de prévoir l'effet de la vitesse de rotation sur l'accroissement des niveaux de bruit ; puisque $\Delta H$ est proportionnel à $\Omega^{2}$ et $Q$ proportionnel à $\Omega$, on trouve dans les deux cas une loi en $\Omega^{4}$ qui correspond à un accroissement de $12 \mathrm{~dB}$ lorsque l'on double la vitesse de rotation.

\section{Etude expérimentale du circuit de pompage}

L'étude du circuit représenté figure 2 a été conduite sur la plate-forme d'essais hydrauliques du CETIM à Nantes ; elle a été effectuée sur une installation comportant les éléments suivants :

- pompe centrifuge à volute simple, à vitesse variable entre 1400 et $3400 \mathrm{tr} / \mathrm{mn}$ développant une puissance hydraulique maximale de $100 \mathrm{~kW}$

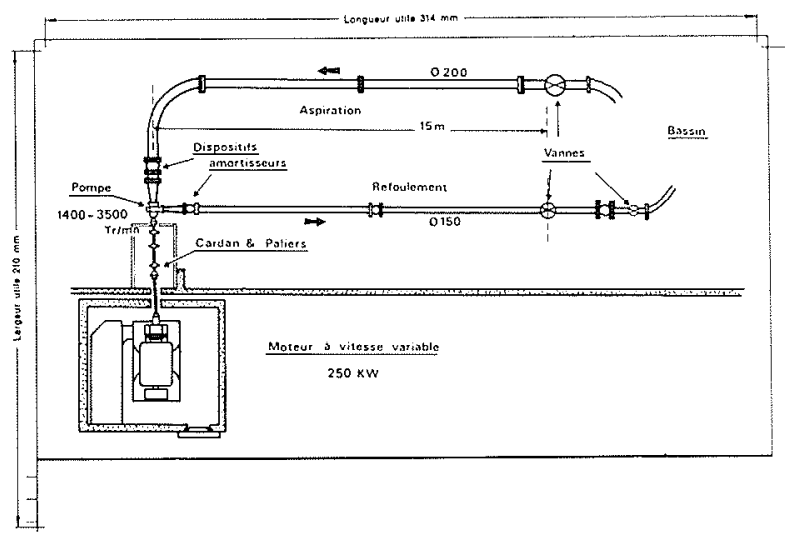

Figure 2 - Schéma du circuit de pompage.

- ligne d'aspiration ( $(200 \mathrm{~mm})$ de longueur variable comportant une vanne permettant de créer la perte de charge nécessaire pour faire caviter la pompe

- ligne de refoulement ( $\varnothing 150 \mathrm{~mm}$ ) d'environ 20 mètres de longueur

- une ou plusieurs vannes de contrôle ( $\emptyset 100 \mathrm{~mm})$ pour le réglage du débit.

Considérant que le système d'entraînement constituait une source de bruit bien spécifique, nous nous sommes limités aux problèmes associés au circuit hydraulique en capotant le moteur et la ligne de l'arbre d'entraînement.

\section{Etude préliminaire de certains éléments $d u$ circuit}

\section{Atténuation apportée par les manchettes souples}

Une étude préliminaire a été réalisée afin de rechercher les moyens efficaces permettant de découpler au mieux les différentes sources vibratoires dans le circuit. Elle a permis de tester une dizaine de manchettes en caoutchouc ou compensateurs de dilatation et elle a mis en évidence que l'insertion de ces systèmes entre deux 
éléments de tuyauterie peut apporter une atténuation de 10 à $30 \mathrm{~dB}$ sur les niveaux de vibration de ces éléments [7].

\section{Bruit des vannes de contrôle}

La vanne de réglage du débit, située au refoulement de la pompe constituant souvent la source de bruit prédominante du circuit, des mesures ont été réalisées sur 2 types de vannes (à double siège et à passage intégral) de diamètre $100 \mathrm{~mm}$, afin de mieux connaître leur comportement acoustique lorsqu'elles sont utilisées dans le circuit. Les essais hydrauliques ont été réalisés en limitant la contribution acoustique de la pompe (fig. 3). On a tracé les caractéristiques hydrauliques (perte de charge, débit) pour 5 ouvertures différentes des clapets de chaque vanne ; la pression amont est maintenue constante par variation de la vitesse de la pompe, le réglage de la pression aval étant effectué par deux autres vannes [4]. On a reporté sur les caractéristiques des vannes les

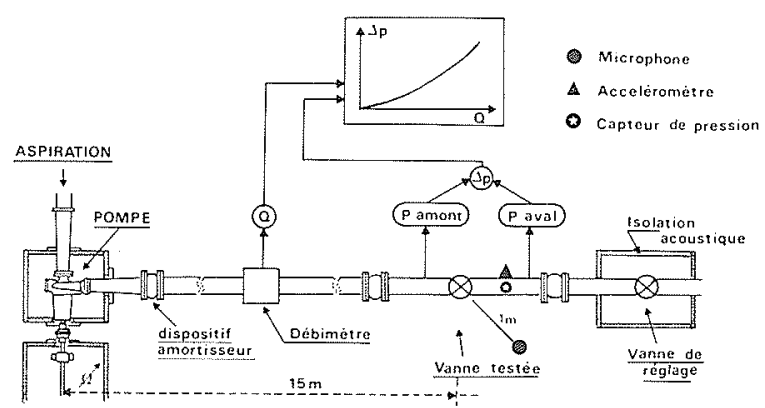

Figure 3 - Montage permettant la détermination parallèle des caractéristiques hydrauliques et acoustiques d'une vanne : - la pression amont est réglée par variation de la vitesse de rotation de la pompe

- la pression aval est contrôlée par une autre vanne silencieuse.

courbes de même niveau de bruit (fig. 4 et 5). Elles montrent que pour des exigences de réglage identiques, ces deux vannes ont un comportement acoustique très différent. La vanne double-siège est surtout bruyante à faible ouverture alors que la vanne à passage direct a un rayonnement maximum lorsque 1'on approche la saturation. Ces remarques ont été utilisées pour le réglage des deux vannes montées en série dans le circuit de refoulement.

Le bruit est essentiellement rayonné par le tronçon de tuyauterie situé immédiatement à l'aval de la vanne, mais une partie non négligeable des excitations est transmise à l'amont par la structure. Pour les essais de la pompe, ce transfert a été limité par l'insertion de manchons.

\section{Etude de la pompe}

Les essais concernant la pompe n'ont pas pour but essentiel la détermination des niveaux globaux absolus de bruit ou de fluctuations de pression mais plutôt une étude des lois d'évolution des bruits aériens et hydrauliques dans chaque domaine de fréquence avec les paramètres de fonctionnement de la pompe (vitesse de rotation, débit, hauteur manométrique, N.P.S.H.) et
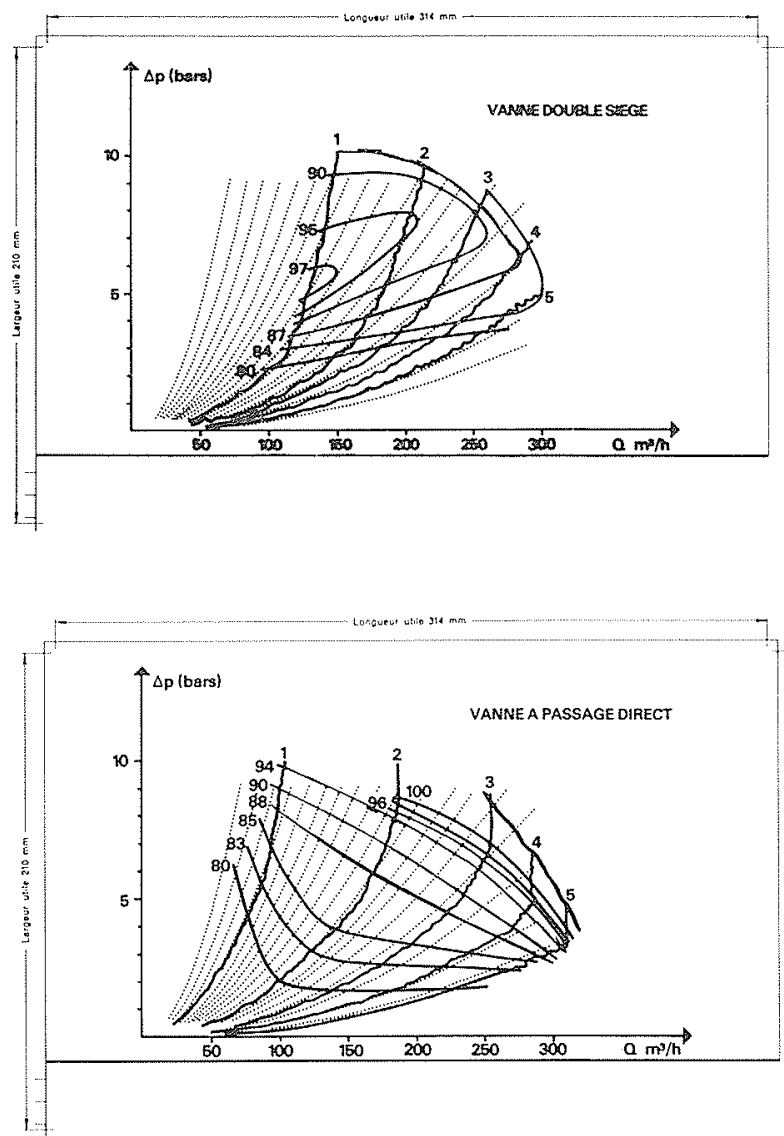

Figures 4 et 5 - Variation du niveau de bruit en fonction des caractéristiques hydrauliques des vannes.

pour différents diamètres de l'impulseur. La principale série d'essais a été effectuée avec un impulseur de diamètre maximum ( $\phi 260 \mathrm{~mm}$ ) comportant cinq aubes.

Il a d'abord été réalisé des mesures globales de bruit aérien (exprimé en $\mathrm{dBA}$ ) relatives à différents points des caractéristiques hydrauliques (hauteur manométrique, débit) correspondant à différentes vitesses de rotation de la pompe (fig. 6). Le réseau de courbes isosoniques tracé permet de prévoir sensiblement le niveau de bruit de la machine pour tout point de fonctionnement.

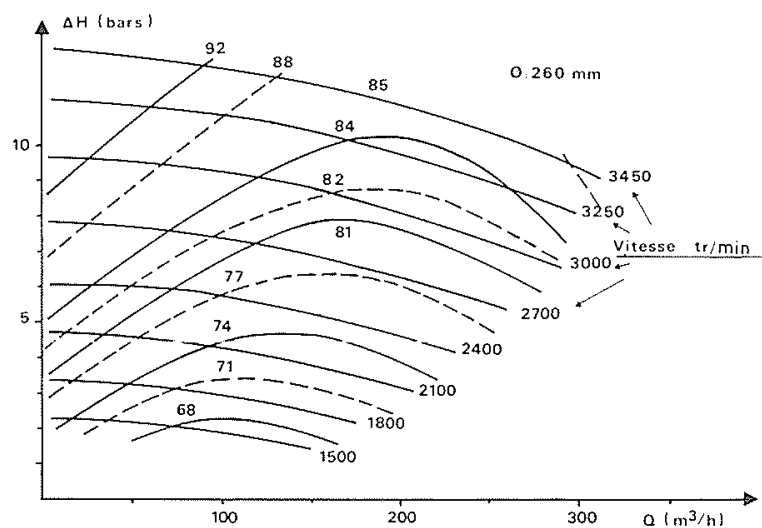

Figure 6 - Courbe de même niveau de bruit d'une pompe centrifuge à vitesse variable en fonction de ses caractéristiques hydrauliques. 


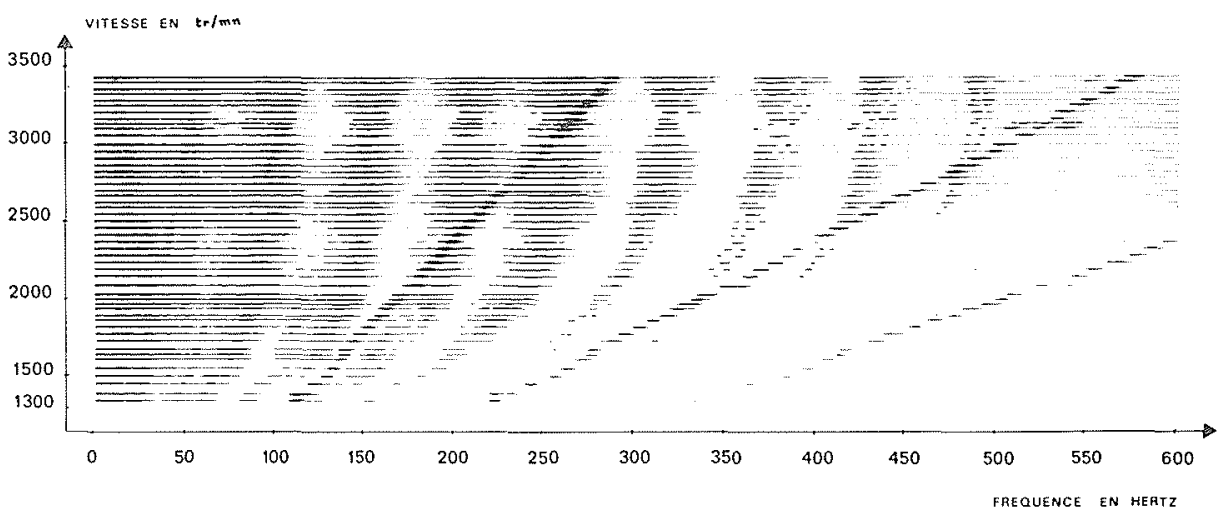

Figure $?$

\section{Evolution des phénomènes en fonction de la vitesse de rotation}

Cette évolution a été effectuée d'une manière très lente entre 1350 et $3400 \mathrm{tr} / \mathrm{mn}$ pour une charge hydraulique correspondant au rendement optimal à $3000 \mathrm{tr} / \mathrm{mn}$. On peut apprécier à l'aide de sonagrammes [3], l'évolution fréquentielle des différentes grandeurs analysées pour 60 valeurs de la vitesse : le spectre des fluctuations de pression mesurées au refoulement et à l'aspiration montre que l'essentiel de l'énergie est contenu au-dessous de $1 \mathrm{kHz}$; on retrouve dans les spectres les raies correspondant au fondamental de la rotation ; l'explication en est la suivante : l'eau utilisée harmoniques ainsi que des phénomènes de résonance. Les niveaux des pulsations de pression obtenus dans la ligne de refoulement dépassent ceux obtenus dans la ligne d'aspiration d'environ $10 \mathrm{~dB}$ et peuvent atteindre $105 \mathrm{~dB}$ (soit des fluctuations de l'ordre de 0,2 bar) ; on retrouve dans le spectre de bruit aérien les mêmes composantes correspondant aux fréquences pures multiples de la vitesse de rotation ainsi que les phénomènes de résonance hydrauliques.

La figure 7 représente l'évolution au cours de la montée en vitesse des analyses spectrales des fluctuations de pression mesurées dans la ligne de refoulement. Un examen attentif montre que les fréquences propres de la colonne fluide croissent sensiblement avec la vitesse de rotation ; l'explication en est la suivante ; l'eau utilisée sur la plate-forme est légèrement chargée en gaz ce qui lui confère une élasticité supplémentaire qui fait chuter la célérité $\mathrm{du}$ son à des valeurs bien inférieures à celles que l'on mesurerait dans un liquide dégazé. Lorsque la vitesse de rotation augmente, la pression crôt dans la ligne de refoulement, ce qui reconcentre l'air, augmente la célérité du son et par là même, les fréquences propres.

Les mêmes essais ont été effectués en injectant de l'air dans la ligne d'aspiration; ils aboutissent à une diminution des niveaux moyens de fluctuations de pression et des phénomènes de résonances hydrauliques mesurés dans la ligne de refoulement. On constate par contre un accroissement du bruit large bande. Ces constatations s'appliquent également au spectre du bruit aérien. On a porté sur les courbes 8 et 9 l'évolution du niveau global des fluctuations de pression et du niveau de bruit (dBA) avec et sans introduction d'air à l'aspiration: l'évolution du bruit hydraulique suit sensible-

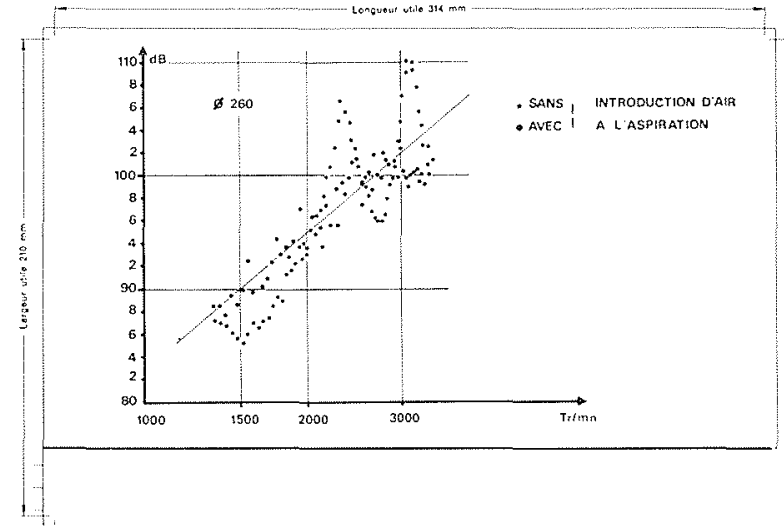

Figure 8 - Evolution du niveau global des fluctuations de pression au refoulement de la pompe.

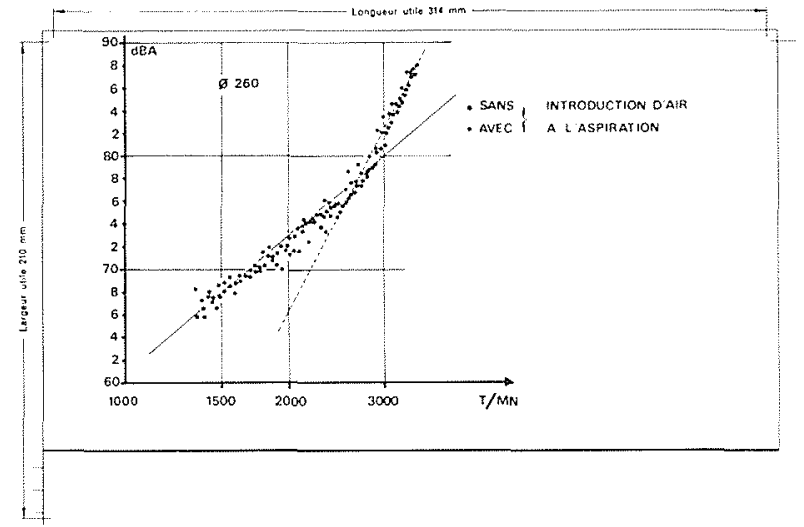

Figure 9 - Evolution du niveau de bruit de la pompe en fonction de la rotation.

ment une loi en $12 \mathrm{~dB} /$ octave lorsqu'il y a introduction d'air ; dans l'autre cas il est plus difficile de conclure en raison des phénomènes de résonance puisque le niveau mesuré dépend de l'emplacement du capteur. Néanmoins le niveau moyen reste assez voisin du précédent. Le fait d'exprimer le bruit aérien en $\mathrm{dBA}$ diminue fortement la contribution des fréquences pures correspondant à la fréquence de passage des aubes qui évolue entre 125 et $300 \mathrm{~Hz}$. Dans les deux cas l'évolution suit sensiblement d'abord une loi en $12 \mathrm{~dB} /$ octave puis au-delà de 3000 tours/mn une loi en plus de $24 \mathrm{~dB} /$ octave ce qui correspond à une désadaptation de la roue. 
Cette détermination expérimentale confirme assez bien les relations prévisionnelles de [5] qui montrent que pour une machine donnée les niveaux de bruit hydraulique et aérien varient comme $10 \log Q \Delta H \Omega$ c'est-à-dire comme $10 \log \Omega^{4}$ (loi en $12 \mathrm{~dB} /$ octave).

\section{Evolution des phénomèrês vibratoires au cours de varia-} tions du débit.

Une fermeture lente de la vanne de contrôle a été effectuée à vitesse constante (2850 tours/mn) jusqu'à un débit très faible.

Les sonagrammes obtenus pour 50 valeurs du débit ont été tracés : ceux qui correspondent aux fluctuations de pression et au bruit aérien montrent que l'énergie vibratoire associée est minimale pour le rendement optimal de la pompe (cas d'une pompe à volute simple). La figure 10 représente l'évolution simultanée du niveau global des pulsations de pression dans la ligne de refoulement, du niveau de bruit (en dBA) et du rendement hydraulique. On constate des variations de plus de $10 \mathrm{dBA}$ pour le bruit aérien et de l'ordre de $7 \mathrm{~dB}$ pour les pulsations de pression.

Les mêmes études effectuées à de plus faibles vitesses montrent que l'écart entre les niveaux mesurés à débit optimum et à débit nul diminuent progressivement avec la vitesse (voir fig. 6).

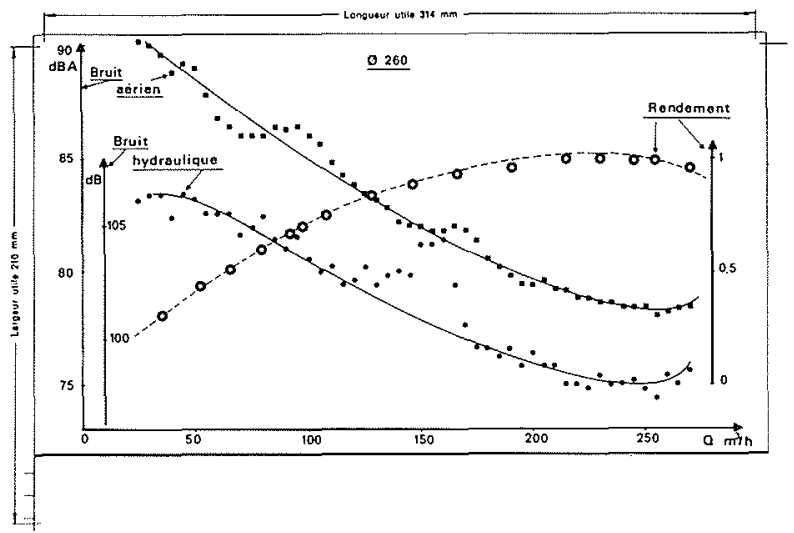

Figure 10 - Evolutions comparées du bruit aérien, du bruit hydraulique et du rendement, en fonction du débit.

Evolution des phénomènes vibratoires avec le N.P.S.H.

Les mesures ont été réalisées à vitesse constante (2850 tours $/ \mathrm{mn}$ ) par réduction très lente de la pression d'aspiration jusqu'à obtention du régime cavitant : les sonagrammes correspondants ont été tracés qui montrent en particulier le développement progressif des composantes hautes fréquences, à la fois pour le bruit hydraulique et le bruit aérien. La figure 11 représente les évolutions simultanées du bruit hydraulique global (dans la ligne d'aspiration), du bruit aérien (exprimé en $\mathrm{dB}$ Lin) et de la puissance hydraulique. On constate un accroissement très important (plus de $7 \mathrm{~dB}$ ) du bruit aérien avant l'altération de $3 \%$ des performances hy. drauliques considéré comme critère de cavitation par les constructeurs de pompe. Une cassure assez nette se remarque dans l'évolution du bruit hydraulique et du bruit

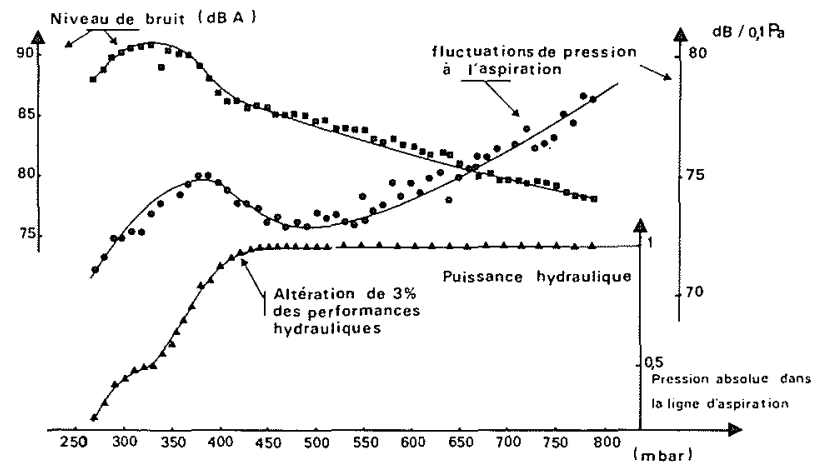

Figure 11 - Evolution des niveaux de bruit et de fluctuations de pression au cours du passage en régime cavitant.

aérien dans cette même zone. Cette constatation a été utilisée depuis pour détecter l'apparition de cavitation sur d'autres machines [4].

Essais effectués avec des impulseurs de différents diamètres

La plupart des essais précédents (variation en vitesse et en débit) ont été réalisés avec 3 autres impulseurs $(\varnothing 240,220,200 \mathrm{~mm}$ ) qui fournissent une puissance hydraulique de plus en plus réduite.

L'examen des sonagrammes montre une réduction progressive du niveau des fluctuations de pression et du bruit aérien à la fréquence de passage des aubes sans toutefois que les niveaux (global pour le bruit hydraulique et global A pour le bruit aérien) soient sensiblement réduits (développement de la turbulence).

Les courbes 6 et 12 montrent que pour une vitesse donnée, le niveau de bruit minimal (exprimé en dBA) obtenu sur une caractéristique ne varie pratiquement pas avec le diamètre de l'impulseur ; par contre à vitesse constante, l'amplitude de la variation avec le débit diminue très sensiblement lorsque le diamètre décroît (à 3000 tours $/ \mathrm{mn}$, plus de $10 \mathrm{~dB}$ pour $\emptyset 260$ et moins de $6 \mathrm{~dB}$ pour $\phi 200$ ). Cette remarque montre que sur le plan acoustique le choix d'un impulseur parfaitement adapté à une volute simple n'est pas à conseiller si la pompe doit fonctionner à des débits très variables.

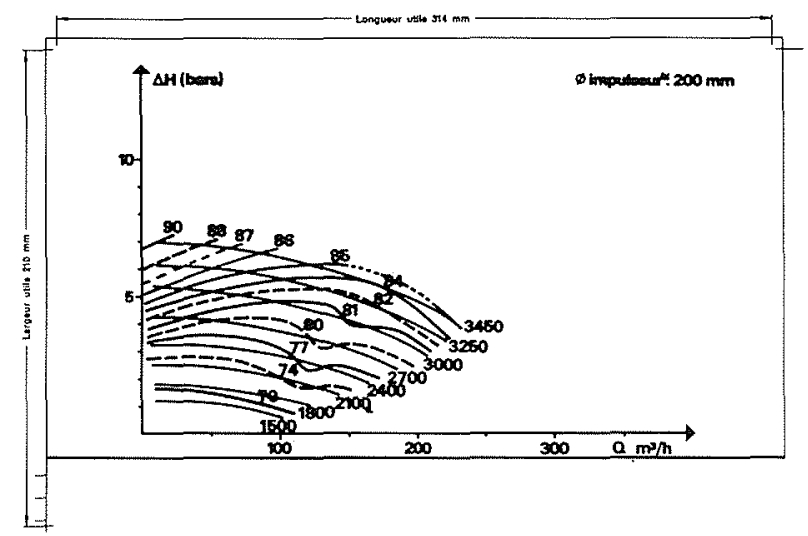

Figure 12 - Variation du niveau de bruit en fonction des caractéristiques hydrauliques de la pompe. 


\section{Transfert d'énergie vibratoire entre le liquide et l'air}

Le rayonnement acoustique de l'ensemble volute de pompe - canalisations dépend essentiellement du niveau des excitations hydrauliques sur les surfaces internes des structures, mais aussi de leur "transparence" à l'énergie vibratoire et de leur aptitude à rayonner.

Des essais globaux ont été réalisés en mesurant d'une part la puissance acoustique de l'installation et d'autre part le niveau (moyenné sur plusieurs capteurs) des fluctuations de pression dans la ligne de refoulement, ceci en faisant varier très fortement en une cinquantaine de paliers les excitations hydrauliques (variation de débit entre débit nul et débit maximum). Il a été montré à l'aide de la méthode sonagramme que, pour les structures considérées, le transfert d'énergie (bruit hydraulique - bruit aérien) s'effectuait de manière privilégiée dans le domaine des moyennes et hautes fréquences (au-delà de $500 \mathrm{~Hz}$ ) ; de plus, le transfert calculé dans chaque bande de tiers d'octave, reste constant avec un écart type de l'ordre de $3 \mathrm{~dB}$ mesuré pour plus de 100 configurations différentes d'excitation. La connaissance des niveaux d'excitations hydrauliques ajoutée à celle du transfert d'énergie par les canalisations permet dans ce cas d'appréhender le rayonnement acoustique de l'installation.

\section{Conclusion}

La réalisation correcte des mesures de bruit rayonné par les pompes centrifuges insérées dans une boucle d'essais nécessite un capotage et un découplage du système d'entraînement et des vannes de réglage.

$\mathrm{Si}$ le niveau de bruit est évidemment lié à la vitesse de rotation, il dépend aussi fortement du point de fonctionnement hydraulique de la machine ainsi que du
N.P.S.H. et des résonances possibles de colonnes liquides dans les tuyauteries ; une définition précise des conditions d'essais est donc nécessaire avant l'exécution des mesures.

L'étude des pulsations de pression délivrées par la pompe fournit des indications intéressantes sur les mécanismes hydrauliques de générations de bruit et renseigne également sur les niveaux des excitations possibles du réseau de canalisations connecté à la machine ; c'est pourquoi il apparaît que parallèlement à la caractérisation d'une pompe par son rayonnement acoustique il est intéressant de la considérer comme source de fluctuations de pression.

L'étude des lois d'évolution du bruit aérien et des fluctuations de pression en fonction de la vitesse de rotation et du débit permet, à partir d'essais réduits la prévision du comportement acoustique de la machine à différents régimes.

\section{Références}

[1] Les bruits industriels : $\mathrm{N}^{\circ}$ Spécial CETIM-Informations, Octobre 1978.

[2] MULLER H.W. - Le bruit des machines volumétriques, V.D.I. Berichte, $\mathrm{n}^{\circ} 228-1975$.

[3] SONAG. - Tiré-à-part CETIM-Informations, $n^{\circ} 41$, Juin 1975.

[4] TOURRET J. - Les bruits hydrauliques industriels, Revue Générale de Thermique, $\mathrm{n}^{\circ}$ 176-177, Août 1976.

[5] SIMPSON H.C., CLARK T.A. - A theoretical investigation of hydraulic noise in pumps, Journal of Sound and Vibration, vol. 5-3 1967.

[6] REID I.Y. - Some factors in the estimation and measurement of airborne noise from centrifugal pumps, Conference I.M.E., Southampton, Sept. 1975.

[7] TOURRET J. - Colloque Européen d'Acoustique FASE 75, Paris. 


\title{
Discussion
}

\author{
Président : M.J. MATTEI
}

Sur proposition du Président de Séance, M.J. MATTEI, la discussion du mémoire de M. TOURRET a été jointe à celle du mémoire de M. BERHAULT, ci-après.

$$
\text { ** } *
$$

Le Président remercie M. TOURRET et M. BERHAULT. 11 ouvre la discussion en rappelant qu'elle portera simultanément sur les deux exposés.

Mme VUONG $(*)$. - Afin de déterminer, par le calcul, le bruit de fonctionnement d'une pompe, nous avons fait une approche théorique en nous basant sur les équations de la conservation de la quantité de mouvement et de masse dans la forme particulière qui leur a été donnée dans les travaux de Monsieur le Professeur FORTIER dans son étude sur les écoulements non stationnaires dans les conduits. En tenant compte du fait que les paramètres $p, q, K, k$ représentant la pression, le débit et les caractéristiques du circuit sont des grandeurs instantanées, fonctions aléatoires du temps, et en considérant leurs expressions adimensionnelles dans l'espace fréquentiel, nous avons obtenu le système d'équation:

$$
\begin{aligned}
& P_{3}^{*}\left(\omega^{*}\right)-P_{2}^{*}\left(\omega^{*}\right)=\frac{\rho \bar{q}^{2} \bar{K}}{S^{2} \Delta_{p}} Q^{*}\left(\omega^{*}\right)+\frac{j \bar{k} \bar{\rho} \bar{q} \omega_{0}}{l \Delta_{p}} \omega^{*} Q^{*}\left(\omega^{*}\right) \\
& +\frac{\rho \bar{q}^{2}}{2 S^{2} \Delta_{p}} K^{*}\left(\omega^{*}\right)-\frac{j \rho \bar{q} \omega_{0}}{l \Delta_{p}} \omega^{*} k^{*}\left(\omega^{*}\right) \\
& Q_{1}^{*}\left(\omega^{*}\right)-Q_{2}^{*}\left(\omega^{*}\right)=\frac{j V \Delta_{p} \omega_{0}}{\rho c^{2} \bar{q}} \omega^{*} P^{*}\left(\omega^{*}\right),
\end{aligned}
$$

En admettant que $Q_{1}^{*}\left(\omega^{*}\right)<<Q_{2}^{*}\left(\omega^{*}\right)$ et $Q_{2}^{* *}\left(\omega^{*}\right) \simeq Q^{*}\left(\omega^{*}\right)$ et sachant que $P_{1}^{*}\left(\omega^{*}\right)-P_{2}^{*}\left(\omega^{*}\right)<<<P^{*}\left(\omega^{*}\right)$ nous avons abouti à une formulation structurale du bruit faisant apparaître le rapport de la fonction spectrale $K^{*}\left(\omega^{*}\right)$ caractéristique du champ de vitesse et de la fonction spectrale $P *\left(\omega^{*}\right)$ caractéristique du champ de pression donnant une image acoustique de la pompe et qui nous conduit à la relation donnant la fluctuation de pression $p^{n}$

$$
p^{0}=\mathrm{A}\left(\frac{\bar{k} V \omega_{0}^{2}}{l c^{2}}\right)^{n}\left(\frac{\rho \bar{q}^{2}}{S^{2} \Delta_{p}}\right)^{m} \Delta_{p} .
$$

dont nous déterminons le cœfficient $A$ et les exposants $n$ et $m$ à partir de mesures expérimentales.

$V$ étant le volume du fluide, $c$ la vitesse du son, $\omega_{0}=2 \bar{n} f_{0}$, $f_{0}$ la fréquence de rotation ou passage des pales, $l$ et $S$ des longueurs et surfaces de référence.

M. BONNIN. - La méthode théorique exposée brièvement par Mme VUONG permet-elle aussi bien d'évaluer la source de bruit pour les divers systèmes actifs (pompes centrifuges ou pompes à déplacement positif) que pour les systèmes passifs (vannes, diaphragmes, singulariés diverses) ?

Quelles sont les difficultés d'application de cette méthode?

Mme VUONG. - La formule que nous avons étudiée est générale. Dans un écoulement instationnaire, nous trouvons les fluctuations de pression à l'origine du bruit. L'équation que nous avons présentée permet de calculer cette fluctuation de pression et ne fait intervenir que des paramètres simples tels que le vo-

(*) Cette intervention a fait l'objet d'une note plus détaillée qui est à la disposition des lecteurs intéressés (S.H.F. - 199, Rue de Grenelle, 75007 Paris). lume du fluide, les surfaces de référence telles que la section à travers laquelle s'écoule le fluide, le débit, la pression utile, la vitesse de rotation. . . Je pense donc qu'on doit pouvoir l'utiliser pour des types de pompes autres que les pompes centrifuges. I suffirait de déterminer la valeur du cœfficient $A$ et des exposants $m$ et $n$.

M. CANA VELIS. - Les problèmes abordés par M. TOURRET revêtent actuellement une importance capitale pour le constructeur de pompes qui doit de plus en plus prévoir et garantir les niveaux de bruits émis par le matériel qu'il founit. Il nous semble qu'un tel sujet mériterait que la S.H.F. organise des conférences uniquement axées sur ce thème.

Le Président. - Je crois que tous les acousticiens se réjouiraient de voir la Société Hydrotechnique de France aborder ces problèmes de façon plus approfondie.

M. BERGERON. - Je crois pouvoir dire, au nom de la Société Hydrotechnique de France que nous enregistrons avec beaucoup d'intérêt cette suggestion. Peut-être conviendrait-il de créer un Groupe de Travail rassemblant quelques spécialistes de la question.

M. GUITON. - Ma première temarque concerne la figure 12 de M. TOURRET (reprise ci-jointe).

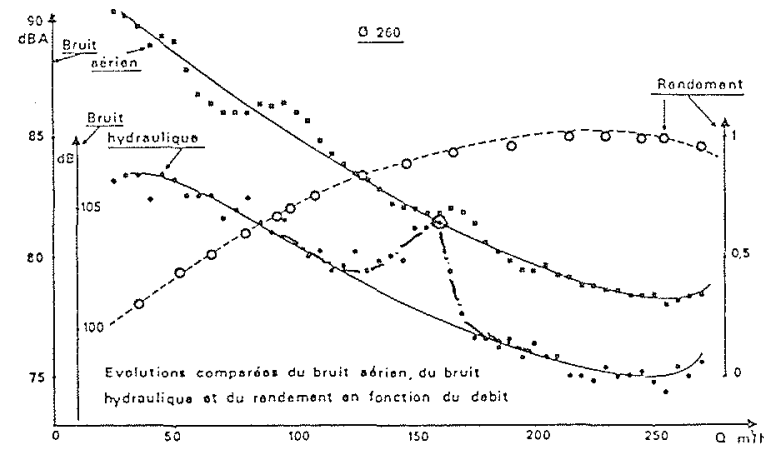

Les points ronds noirs, représentatifs du bruit hydraulique en fonction du débit montrent un pic aux alentours du débit $160 \mathrm{~m}^{3} / \mathrm{h}$. (Le rendement maximum étant vers $225 \mathrm{~m}^{3} / \mathrm{h}$ ).

Ce pic me semble assez caractéristique du débit critique limitant la zone des débits partiels étudiés par le Groupe de Travail $n^{\circ} 1$ de la S.H.F. Un tel pic se présente du reste aussi pour d'autres grandeurs que le bruit hydraulique, influencées elles aussi par ce débit critique.

Dans bien des cas, comme cela a été fait sur la courbe en trait plein de la Figure 12, on a tendance à faire disparaitre ces pics en "lissant" les courbes. Ici, entre $125 \mathrm{~m}^{3} / \mathrm{h}$ et $175 \mathrm{~m}^{3} / \mathrm{h}$, il vaudrait mieux la courbe que je fais figurer en traits mixtes et qui montre bien le pic, plutôt que la courbe noire qui passe "en tunnel", sans aucun point, sous ce pic.

$M$. TOURRET. - Le bruit hydraulique dont l'évolution irrégulière vient d'être signalée par M. GUITON aux environs de $160 \mathrm{~m}^{3} / \mathrm{h}$ correspond au signal global mesuré au refoulemerít de la pompe équipée de la roue de diamètre maximum $(\emptyset)$ 260), la pompe tournant à vitesse constante (2.850 tours/ $\mathrm{mn})$. L'examen plus fin des sonogrammes réalisées en $1 / 3$ octave et en analyse en bande étroite montre que l'accroissement du niveau global résulte essentiellement de l'accroissement des composantes périodiques (harmoniques 3 et 6 de la vitesse de rotation alors que le nombre d'aubes est égal à 5) ; ce phénomène se retrouve d'ailleurs encore plus marqué à l'aspiration, ce qui exclue une interprétation basée sur la modification des phénomènes de résonance dans la ligne de refoulement associés 
à la fermeture progressive de la vanne double siège contrôlant le débit. Lorsqu'on fonctionne avec les impulseurs de plus faible diamètre $(\varnothing 240,220)$, le phénomène de renforcement se produit également pour un débit chaque fois un peu plus faible, et correspond également à l'accroissement du niveau des composantes périodiques.

M. GUITON. - Ma seconde remarque concerne la figure 7 de M. TOURRET

Cette figure montre bien que lorsqu'une pompe a une large plage de variation de vitesse de rotation, il n'est pas possible d'éviter des zones de coïncidence entre des fréquences liées au passage des aubes de la roue et les diverses fréquences propres du circuit.

C'est le cas en particulier des pompes alimentaires des postes d'eau des centrales nucléaires PWR, postes d'eau dont un schéma a été présenté hier après-midi dans la conmunication de MM. LARMINAUX et OURMANN.

Pendant la montée en puissance du réacteur, on ne balaie que très lentement toute la plage des vitesses de rotation. En outre Ie poste d'eau est un réseau maillé très complexe qui présente de nombreuses fréquences propres. Il est donc nécessaire, pour réduire les résonances, que le niveau des fluctuations de pression excitées par la pompe à sa sortie soit faible.

M. TOURRET. - La remarque formulée est parfaitement valable dans le cas de machines fonctionnant sur une large plage de vitesse : par contre, dans le cas de machines à régime de rotation constant, il peut être intéressant d'éviter les phénomènes de résonances par un calcul prévisionnel. Bien sûr, dans les deux cas, il est souhaitable de réduire les niveaux des fluctuations de pression.

M. CANAVELIS. - M. TOURRET a fait remarquer que la variation du bruit en fonction du débit est moindre pour une roue "rognée" que pour une roue de grand diamètre. Ce phénomène s'explique assez bien par le fait que l'entrefer entre roue et volute est plus grand et que les intéractions entre le sillage des aubes tournantes et le bec de volute diminuent.

M. TOURRET. - Pour une vitesse de rotation donnée de la machine, l'amplitude de la variation du bruit avec le débit diminue très sensiblement lorsque le diamètre décroît (plus de $10 \mathrm{~dB}$ pour $\varnothing 260$ et moins de $6 \mathrm{~dB}$ pour $\emptyset 200$ ).

M. CANAVELIS. - Vous avez dit que lorsque la cavitation apparaît, on voit augmenter l'énergie spectrale dans les moyennes et hautes fréquences. Je n'ai pas eu entre les mains le texte de la conférence de MM. de LOOF et LEDUCQ, mais je lis dans le résumé : quand il y a cavitation, la majeure partie de l'énergie se situe dans la partie en basse fréquence $(f \leqslant 200 \mathrm{~Hz})$. J'aimerais comprendre pourquoi.

M. TOURRET. - Comme nous l'avons vu, le bruit hydraulique en régime non cavitant est essentiellement concentré dans les fréquences inférieures à $1 \mathrm{kHz}$ et le niveau est généralement très faible au-delà de $10 \mathrm{kHz}$. Lorsqu'il y a apparition de cavitation, l'énergie libérée se répartit sur une gamme considérable de fréquences (d'une centaine à plusieurs centaines de $\mathrm{kHz}$ ) ; l'accroissement relatif est généralement faible dans les basses fréquences où le "bruit de fond" est important alors qu'il peut être très supérieur pour des fréquences telles que $40 \mathrm{kHz}$.

En pratique, pour détecter l'apparition de cavitation on cale un filtre passe bande centré par exemple sur $40 \mathrm{kHz}$, et l'on étudie l'évolution du niveau de bruit ainsi filtré.

$M . L E D U C Q$. - On se place en hautes fréquences pour détecter la cavitation par moyen acoustique, parce qu'à ces fréquences l'augmentation relative de niveau de bruit est très importante. On fait en quelque sorte une détection par tout ou rien.

En fait, si on pouvait isoler la cavitation de son contexte hydrodynamique, on aurait un phénomène avec spectre à très large bande, excitant à son tour des "résonances" dans le fluide, d'où une augmentation d'ensemble sur toute la largeur du spectre.

M. CANAVELIS. - Ma dernière remarque s'adresse à MM. BERHAULT et TOURRET. Est-ce que M. TOURRET pense qu'on peut, avec la démarche de $M$. BERHAULT. en appliquant des excitateurs sur des corps de pompes industrielles, trouver l'impédance mécanique de la machine et trouver la fonction de transfert?

M. BERHAULT. - Bien sûr, c'est une méthode très générale en hydraulique. Je l'ai utilisée moi-même pour déterminer des fréquences de résonance de très grosses vannes pour des centrales nuctéaires pour des questions sismiques. On peut, avec ce matériel, faire des mesures très complètes.

M. TOURRET. - Un travail similaire a été conduit sur une volute de pompe centrifuge assez épaisse $(3 \mathrm{~cm})$ et a permis de mettre en évidence les modes de vibration de la structure dans des gammes de fréquences allant de $500 \mathrm{~Hz}$ à plusieurs $\mathrm{kHz}$. Il s'agit d'une méthode d'excitation par chocs (en une centaine de points) avec mesure de réponse en un seul point et détermination des déformés par calcul.

M. BERHAULT. - En dehors de la méthode de balayage en fréquence, qui donne de bons résultats, on peut utiliser l'excitation par chocs. Quelqu'un a parlé hier d'un essai en conduite ; c'est la même technique ; c'est un peu plus systématique dans notre cas. Cela donne seulement des ordres de grandeur mais permet de voir s'il $\mathrm{y}$ a de graves problèmes de résonance dans la gamme sensible. En acoustique, entre 500 et $20 \mathrm{kHz}$. il y a des excitations assez importantes. On peut s'arranger pour les faire glisser d'un côté ou de l'autre dans les hautes fréquences.

M. TOURRET. - La connaissance des déformés constitue un premier point, mais il est également nécessaire de déterminer la répartition des efforts dynamiques (fluctuations de pression, sollicitations au niveau des paliers) afin d'obtenir la réponse globale de la structure et agir sur celle-ci ; ainsi, on a pu mettre en évidence que pour certains modes de vibration, le bec de volute peut être le siège de déformation importantes sous l'effet d'efforts périodiques associés au passage des aubes de la roue.

Le Président. - Je remercie $\mathrm{M}$. TOURRET et $\mathrm{M}$. BERHAULT. 